\title{
Clinical Phenotype of Japanese Patients with Dermatomyositis —Classification Based on Dermatomyositis-Specific Autoantibodies
}

\author{
Shinji Sato ${ }^{*}$, Masataka Kuwana ${ }^{2}$, Michito Hirakata², Shinichi Nogi ${ }^{1}$, Noriko Sasaki', \\ Naofumi Chinen', Kiri Honda1, Eiko Saito', Takayuki Wakabayashi', Chiho Yamada1, \\ Yasuo Suzuki ${ }^{1}$ \\ ${ }^{1}$ Division of Rheumatology, Department of Internal Medicine, Tokai University, School of Medicine, \\ Isehara, Japan \\ ${ }^{2}$ Division of Rheumatology, Department of Internal Medicine, Keio University, School of Medicine, \\ Tokyo, Japan \\ Email: shinsjam@tokai-u.jp
}

Received 5 April 2014; revised 5 May 2014; accepted 12 May 2014

Copyright (C) 2014 by authors and Scientific Research Publishing Inc.

This work is licensed under the Creative Commons Attribution International License (CC BY).

http://creativecommons.org/licenses/by/4.0/

c) (i) Open Access

\section{Abstract}

Objectives: To correlate the precise specificity of autoantibodies in Japanese dermatomyositis (DM) patients with their clinical phenotypes. Methods: Serum samples from 94 adult DM patients (67 with classical DM and 27 with clinically amyopathic dermatomyositis, CADM) were screened for autoantibodies using immunoprecipitation assays. Patients with antibodies against aminoacyl transfer RNA synthetase (ARS), Mi-2 or who had other autoantibodies were assessed for clinical symptoms and laboratory findings. Results: Sera from 27 of $94 \mathrm{DM}$ patients (29\%) were found to have anti-ARS antibodies. Nineteen (20\%) had anti-CADM-140/MDA5, 5 (5\%) had anti-Mi-2, and 8 (6\%) had anti-p155/TIF1- $\gamma$. Anti-MJ/NXP-2 was not found in our series of adult DM. Seventeen patients with anti-ARS had fever and 22 had arthritis and interstitial lung disease (ILD), compatible with a diagnosis of anti-ARS syndrome. Seventeen of 19 (89\%) with anti-CADM140/MDA5 had ILD, 16 (84\%) of whom developed rapidly progressive ILD (RP-ILD). Four of 5 $(80 \%)$ with anti-Mi-2 had heliotrope rash and/or Gottron's sign/papules, and $2(40 \%)$ had V-sign and/or shawl-sign rash, whereas no ILD or malignancy was detected. As seen with anti-Mi-2positive patients, a low frequency of ILD (13\%) was found in patients with anti-p155/TIF1- $\gamma$ but 6 of $8(75 \%)$ had malignancy during their course. The frequency of ILD was significantly higher in patients with anti-ARS or anti-CADM-140/MDA5 compared with anti-Mi-2 or anti-p155/TIF1- $\gamma$ (81\% and 89\%, respectively). It should be noted that anti-CADM-140/MDA5-positive patients suffered significantly more RP-ILD compared to patients with anti-ARS $(84 \%$ vs. $7 \%, P<0.0001)$. On the other hand, anti-p155/TIF1- $\gamma$ positive patients had a significantly higher rate of ma-

${ }^{*}$ Corresponding author. 
lignancy compared with anti-ARS-, anti-CADM-140/MDA5- and anti-Mi-2-positive patients (75\% vs. 7\%: $P=0.0004,5 \%: P=0.0006,0 \%: P=0.02$, respectively). Conclusions: These results indicate that in addition to antibodies previously identified as specific for DM, autoantibodies newly found in these patients are useful for stratifying them into clinical subgroups.

\title{
Keywords
}

\author{
Dermatomyositis (DM), Interstitial lung Disease (ILD), Rapidly Progressive Interstitial \\ Lung Disease (RP-ILD), Malignancy, Myositis-Specific Autoantibodies
}

\section{Introduction}

Dermatomyositis (DM) is a chronic inflammatory disorder that affects skin and muscle and is often accompanied by interstitial lung disease (ILD) or malignancy [1] [2]. The clinical manifestations are diverse in individual patients, reflecting subtypes of DM. Autoantibodies directed against aminoacyl transfer RNA synthetases (ARS), signal recognition particle (SRP), and Mi-2 are specific markers of polymyositis (PM)/DM [3] [4]. These wellknown autoantibodies have proven clinically useful for the diagnosis and classification of PM/DM patients into subtypes and for predicting the response to treatment and prognosis.

Recently, new DM-specific autoantibodies have been discovered. Targoff et al. and Kaji et al. reported the presence of autoantibodies against a $155 \mathrm{kDa}$ protein (anti-p155/TIF1- $\gamma$ (transcriptional intermediary factor 1- $\gamma$ antibodies) in patients with DM and found that they are closely associated with malignancy [5] [6]. We have identified autoantibodies against a $140 \mathrm{kDa}$ protein (anti-CADM-140/MDA5 (melanoma differentiation-associated gene 5) antibodies) mainly in patients with clinically amyopathic dermatomyositis (CADM) [7] [8].

Although some clinical characteristics of Japanese DM patients with these newly-identified antibodies have been reported, thus far, correlations between the pattern of autoantibodies present and the patient's clinical course have been imprecise. In the present study, we investigated the variety of autoantibodies in Japanese DM patients in relation to their specificity and association with clinical phenotypes.

\section{Methods}

\subsection{Patients and Sera}

Serum samples from 94 Japanese patients with adult DM (67 with classical DM and 27 with CADM) who were seen at Keio University Hospital (2001-2008) and Tokai University Hospital (2010-2013) were screened for autoantibodies using immunoprecipitation (IP) assays. Blood samples were obtained after the patients had provided written informed consent approved by the Keio and Tokai University Institutional Review Boards.

\subsection{Immunoprecipitation (IP)}

The IP assay with K562 or HeLa cell extracts was performed as previously described [9]. For analysis of RNAs, antibodies bound to protein A-Sepharose CL-4B beads (Pharmacia Biotech AB, Uppsala, Sweden) were incubated with extracts of K562 or HeLa cells. They were then washed with NET-2 buffer (50 mM Tris-HCL, pH 7.5, $150 \mathrm{mM} \mathrm{NaCl}$ (Wako Pure Chemical Industries, Osaka, Japan), 0.05\% Nonidet P-40 (Nacalai tesque, Kyoto, Japan). After ethanol precipitation, RNAs were dissolved in electrophoresis sample buffer composed of $10 \mathrm{M}$ urea (Bio-Rad Laboratories, Hercules, CA), 0.025\% bromophenol blue (Wako Pure Chemical Industries, Osaka, Japan), and 0.025\% xylene cyanol-FF (Wako Pure Chemical Industries, Osaka, Japan) in TBE buffer (90 mM Tris-HCl, pH 8.6, $90 \mathrm{mM}$ boric acid (Wako Pure Chemical Industries, Osaka, Japan), and $1 \mathrm{mM}$ EDTA (Wako Pure Chemical Industries, Osaka, Japan)). The RNA samples were resolved in $7 \mathrm{M}$ urea-10\% polyacrylamide gels, which were then silver stained (Bio-Rad Laboratories, Hercules, CA). For protein studies, antibody-coated Sepharose beads were mixed with $\left[{ }^{35} \mathrm{~S}\right]$ methionine-labeled K562 or HeLa extracts. After washing, the Sepharose beads were resuspended in SDS-sample buffer (2\% SDS (Sigma, St. Louis, USA), 10\% glycerol (Wako Pure Chemical Industries, Osaka, Japan), $62.5 \mathrm{mM}$ Tris-HCl, pH 6.8, 0.005\% bromophenol blue). The proteins were then fractionated by $10 \%$ SDS-PAGE and dried. Radiolabeled protein components were analyzed by autora- 
diography. Anti-ARS, anti-SRP, anti-Mi-2, known myositis-specific antibodies, as well as anti-CADM-140/MDA5 and anti-p155/TIF1- $\gamma$ are detectable with these assays by comparison with corresponding standard sera [10].

\subsection{Clinical Features}

The clinical symptoms, clinical course, and laboratory findings were retrospectively assessed from medical records in all patients including those positive for anti-ARS, anti-Mi-2, anti-CADM-140/MDA5 and anti-p155/ TIF1- $\gamma$ autoantibodies.

The diagnosis of DM was based on the diagnostic criteria proposed by Bohan and Peter [11]. The assessment of muscle weakness was performed according to a manual muscle test (MMT) [12]. The diagnosis of CADM was based on classification criteria proposed by Sontheimer et al. [13], i.e. DM patients with no clinical muscle symptoms for more than two years after the onset of skin manifestations. The remission of myositis was defined as both improvement of muscle strength on MMT and normalization of the serum creatine kinase (CK) value.

ILD was defined based on findings of chest radiography, chest CT, and lung function testing. Rapidly progressive ILD (RP-ILD) was defined as a subset of patients presenting progressive dyspnea along with hypoxemia and worsening of interstitial changes on the chest X-ray within one month of the onset of respiratory symptoms.

\subsection{Statistical Analysis}

All comparisons between each patient group were performed using Fisher's 2-tailed exact test or Student's t-test. Two-sided p-values of less than 0.05 were considered statistically significant.

\section{Results}

\subsection{Detection of Autoantibodies in Patients with DM}

Using IP, we screened 94 DM patients'sera including 67 patients with classical DM and 27 with CADM. Myositis-specific autoantibodies (anti-ARS and anti-Mi-2) and myositis-associated autoantibodies (anti-SSA/Ro, anti-U1RNP) as well as recently identified autoantibodies (anti-CADM-140/MDA5, anti-p155/TIF1- $\gamma$ ) were studied. These proteins were easily distinguishable from those immunoprecipitated by sera reactive with other known antigens [14].

\subsection{Frequencies of Autoantibodies in Patients with DM (Table 1)}

Sera from 27 of the 94 DM patients (29\%, 25 with classical DM and 2 with CADM) were found to contain

Table 1. Frequencies of autoantibodies in patients with DM.

\begin{tabular}{|cccc|}
\hline & Dermatomyositis & & \\
\hline Autoantibodies $n$ (\%) & Classical DM & CADM & Total \\
\hline Myositis-specific antibodies & $\mathrm{N}=67$ & $\mathrm{~N}=27$ & $\mathrm{~N}=94$ \\
Anti-ARS & & & \\
Anti-SRP & $25(37)$ & $2(7)$ & $27(29)$ \\
Anti-Mi-2 & $0(0)$ & $0(0)$ & $0(0)$ \\
\hline Anti-SSA/Ro & $5(7)$ & $0(0)$ & $5(5)$ \\
Anti-U1RNP & & & $10(11)$ \\
Myositis-associated antibodies & $8(12)$ & $2(7)$ & $4(4)$ \\
Newly found DM-specific antibodies & $4(6)$ & $0(0)$ & $19(20)$ \\
Anti-CADM-140/MDA5 & & $17(63)$ & $8(9)$ \\
Anti-p155/TIF1- $\gamma$ & $2(3)$ & $1(4)$ & $0(0)$ \\
Anti-MJ/NXP-2 & $7(10)$ & $0(0)$ & $36(38)$ \\
Negative & $0(0)$ & $6(22)$ & \\
\hline
\end{tabular}

DM: dermatomyositis, CADM; clinically amyopathic dermatomyositis, ARS: aminoacyl tRNA sythetase, SRP: signal recognition particle. 
anti-ARS antibodies (anti-Jo-1: 10; anti-EJ: 7; anti-PL-7: 5; anti-PL-12: 4; anti-OJ: 1). Five (5\%) with classical DM had anti-Mi-2, 19 (20\%, 2 with classical DM and 17 with CADM) had anti-CADM-140/MDA5, and 8 (9\%, 7 with classical DM and 1 with CADM) had anti-p155/TIF1- $\gamma$ antibodies. Interestingly, one patient with classical DM had both anti-Jo-1 and anti-p155/TIF1- $\gamma$ antibodies. Anti-MJ/NXP-2 was not found in our series of adult DM; neither was anti-SRP, or PM-specific antibodies detected. Thus, overall, 58 of the 94 (62\%) DM patients had myositis-specific antibodies.

\subsection{Clinical Characteristics in Patients According to Possession of Each Autoantibody (Table 2)}

\subsubsection{Anti-ARS Antibodies}

Of the anti-ARS antibody-positive patients, 25 had classical DM and 2 CADM. Anti-Jo- 1 was the antibody most frequently present, followed by anti-EJ. Most patients had fever (63\%), arthritis (81\%) and Raynaud's phenomenon (30\%). Twenty-two patients (81\%) had ILD and 20 of these (91\%) had a chronic course. These clinical features are consistent with the anti-ARS syndrome previously proposed.

\subsubsection{Anti-CADM-140/MDA5 Antibodies}

Similar to anti-ARS antibody-positive patients, many of those with anti-CADM-140/MDA5 antibodies had fever (68\%), arthritis (79\%) and ILD (89\%). However, in contrast to anti-ARS-positive patients, 16 of 17 (94\%) with ILD developed RP-ILD. Treatment with high-dose prednisolone including pulse therapy with intra-venous intermittent cyclophosphamide and/or cyclosporine A was given to all 16 RP-ILD patients, with respiratory symptom improvement in 12 (75\%).

\subsubsection{Anti-Mi-2 Antibodies}

All 5 Mi-2-antibody-positive patients were diagnosed as classical DM with obvious muscle weakness and serum CK elevation. Steroid therapy for muscle symptoms was started and all patients improved. Four of 5 (80\%) had typical DM skin manifestations, heliotrope rash and/or Gottron's sign/papules and 2 (40\%) had V-sign or shawl-sign rash. No ILD or malignancy was detected in any of these patients.

\subsubsection{Anti-p155/TIF1- $\gamma$ Antibodies}

A relatively high frequency of V-sign and/or shawl-sign rash (63\%) and a low frequency of ILD (13\%) were found in patients with anti-p155/TIF1- $\gamma$ antibodies. It is noteworthy that 6 of 8 (75\%) had malignancy (bladder, gastric, lung, ovarian, peritoneal cancer, malignant lymphoma) during their course.

\begin{tabular}{|c|c|c|c|c|c|}
\hline Clinical and laboratory findings & Anti-ARS & Anti-CADM-140/MDA5 & Anti-Mi-2 & Anti-p155/TIF1- $\gamma$ & Negative \\
\hline & $(n=27)$ & $(\mathrm{n}=19)$ & $(n=5)$ & $(\mathrm{n}=8)$ & $(\mathrm{n}=36)$ \\
\hline Age at onset (mean \pm SD) & $52 \pm 14$ & $53 \pm 13$ & $55 \pm 17$ & $62 \pm 20$ & $51 \pm 14$ \\
\hline Male/female & $8 / 19$ & $5 / 14$ & $2 / 3$ & $2 / 6$ & $7 / 29$ \\
\hline Gottron’s sign or papules (\%) & 67 & 84 & 80 & 88 & 78 \\
\hline Heliotrope rash (\%) & 37 & 37 & 80 & 38 & 56 \\
\hline V-sign or Shawl-sign (\%) & 15 & 37 & 40 & 63 & 31 \\
\hline Muscle weakness (\%) & 93 & 11 & 100 & 88 & 81 \\
\hline Elevation of CK (\%) & 93 & 26 & 100 & 88 & 78 \\
\hline Fever (\%) & 63 & 68 & 20 & 25 & 53 \\
\hline Arthritis (\%) & 81 & 79 & 60 & 13 & 50 \\
\hline Raynaud's phenomenon (\%) & 30 & 5 & 0 & 13 & 6 \\
\hline $\operatorname{ILD}(\%)$ & 81 & 89 & 0 & 13 & 50 \\
\hline Rapidly progressive ILD (\%) & 7 & 84 & 0 & 0 & 8 \\
\hline Malignancy (\%) & 7 & 5 & 0 & 75 & 19 \\
\hline ANA (FANA) (\%) & 44 & 16 & 100 & 88 & 36 \\
\hline
\end{tabular}

$\mathrm{CK}=$ creatine kinase; ILD = interstitial lung disease; ANA = anti-nuclear autoantibodies. 


\subsection{Comparison of Clinical Features in Patients Relative to Each Autoantibody}

The frequency of V-sign or shawl-sign rash in patients with anti-p155/TIF1- $\boldsymbol{\gamma}$ was significantly higher than in patients with anti-ARS $(15 \%$ vs. $63 \%$ : $P=0.015)$. The frequency of ILD was significantly higher in patients with anti-ARS or anti-CADM-140/MDA5 in comparison with anti-Mi-2-positive (81\% vs. $0 \%$ : $\mathrm{P}=0.0013$, 89\% vs. $0 \%: \mathrm{P}=0.0005$, respectively) and anti-TIF1- $\gamma$ positive patients $(81 \%$ vs. $13 \%: \mathrm{P}=0.0008,89 \%$ vs. $13 \%: \mathrm{P}=$ 0.003 , respectively). Moreover, it should be noted that anti-CADM-140/MDA5-positive patients suffered significantly more RP-ILD compared to patients with anti-ARS (7\% vs. 84\%, P < 0.0001). Interestingly, anti-p155/TIF1- $\gamma$ positive patients had a significantly higher rate of malignancy than anti-ARS-, anti-CADM140/MDA5- or anti-Mi-2-positive patients (75\% vs. 7\%: $\mathrm{P}=0.0004,5 \%: \mathrm{P}=0.0006,0 \%: \mathrm{P}=0.02$, respectively).

\section{Discussion}

We surveyed autoantibodies in Japanese patients with DM and analyzed the association between clinical characteristics and antibody specificity. We found a close association between clinical features and the presence of each particular autoantibody specificity.

In our series, 58 of 94 (62\%) patients with DM possessed at least one DM-specific antibody (anti-ARS, anti-Mi-2, anti-CADM-140/MDA5 or anti-p155/TIF1- $\gamma$ ). This is a slightly higher rate than in previous reports. Hamaguchi et al. reported the presence of these antibodies in 147 of 376 (39\%) patients [15] and Ikeda et al. found them in 29 of 55 (53\%) patients [16]. Thus, the discovery of new DM-specific antibodies in recent years enables us to classify more than half of all DM patients into specific clinical entity groups and to predict their prognosis. Moreover, these antibodies are useful for making a correct diagnosis in daily clinical practice.

We tried to make the clinical subtyping of DM patients using DM-specific autoantibodies more accurate (Table 3). Eight autoantibodies to ARS have been described so far, the most frequent being anti-Jo-1 antibody. Anti-ARS antibodies are known as being specific for PM/DM patients although some of these antibodies seem to have stronger associations with ILD. Interestingly, these anti-ARS-positive patients had common clinical manifestations such as fever, arthritis, Raynaud's phenomenon, mechanic's hand and chronic ILD. They represent patients with "anti-ARS syndrome". Therefore, anti-ARS autoantibodies are common in classical DM with a high frequency of fever, arthritis, and chronic ILD. On the other hand, anti-CADM-140/MDA5 antibody is more frequently detected in CADM patients especially in Japan. The most striking characteristic of the presence of this antibody is its strong association with RP-ILD, a DM complication with poor prognostic implications. Because it is likely that early diagnosis and treatment is important in this condition, measuring anti-CADM140/MDA5 antibody could significantly benefit DM and RP-ILD patients. Patients with anti-Mi-2 antibody tended to have typical DM skin manifestations (Heliotrope rash or Gottron’s sign and/or papules). Complications that affect their prognosis such as ILD or neoplasia seem to occur with very low frequency. In this respect,

\begin{tabular}{ccccc}
\hline Table 3. Clinical subtyping using DM-specific autoantibodies. & & \\
\hline Clinical and laboratory findings & Anti-ARS & Anti-CADM-140/MDA5 & Anti-Mi-2 & Anti-p155/TIF1- $\gamma$ \\
\hline Typical DM rash & & & $\circ$ & \\
V-sign or Shawl-sign & & $\times$ & $\circ$ & $\circ$ \\
Muscle weakness & 0 & $\times$ & $\circ$ & $\circ$ \\
Elevation of CK & 0 & $\circ$ & $\times$ & $\times$ \\
Fever & 0 & $\circ$ & $\times$ & $\times$ \\
Arthritis & 0 & $\circ$ & $\times$ & $\times$ \\
Rapidly progressive ILD & $\times$ & $\times$ & $\times$ & $\circ$ \\
Chronic ILD & $\circ$ & & $\circ$ & $\circ$ \\
Malignancy & $\times$ & $\times$ & $\times$ \\
ANA (FANA) positive (\%) & & & $\times$ \\
\hline
\end{tabular}

CK = creatine kinase; ILD = interstitial lung disease; ANA = anti-nuclear autoantibodies; $\circ=$ relatively frequent; $\times$ = relatively low frequent or rare. 
patients with anti-Mi-2 antibody have a relatively good prognosis. Finally, anti-p155/TIF1- $\gamma$ is detected in both classical DM and CADM with DM typical skin rash as well as V-sign and/or Shawl-sign. Patients with this antibody have a high frequency of malignancy which should therefore be considered at the time of diagnosis.

\section{Conclusion}

New autoantibodies specifically found in DM as well as previously well-known DM-specific antibodies are useful for classifying DM patients into clinical subgroups as well as for differential diagnosis, choice of treatment and prediction of outcome in daily clinical practice. Further efforts to discover new DM-specific antibodies will facilitate progress in the clinical management of DM.

\section{Disclosures}

None by all authors.

\section{Acknowledgements}

We thank Ms. Mutsuko Ishida, Etsuko Iwata and Miyako Nakagawa for assisting in RNA and protein immunoprecipitation assays.

\section{References}

[1] Targoff, I.N. (2004) Laboratory Testing in the Diagnosis and Management of Idiopathic Inflammatory Myopathies. Rheumatic Disease Clinics of North America, 28, 859-890. http://dx.doi.org/10.1016/S0889-857X(02)00032-7

[2] Mimori, T., Imura, Y., Nakashima, R. and Yoshifuji, H. (2007) Autoantibodies in Idiopathic Inflammatory Myopathy: An Update on Clinical and Pathophysiological Significance. Current Opinion in Rheumatology, 19, 523-529. http://dx.doi.org/10.1097/BOR.0b013e3282f01a8c

[3] Targoff, I.N., Johnson, A.E. and Miller, F.W. (1990) Antibody to Signal Recognition Particle in Polymyositis. Arthritis \& Rheumatology, 33, 1361-1370. http://dx.doi.org/10.1002/art.1780330908

[4] Targoff, I.N. and Reichlin, M. (1985) The Association between Mi-2 Antibodies and Dermatomyositis. Arthritis \& Rheumatology, 28, 796-803. http://dx.doi.org/10.1002/art.1780280711

[5] Targoff, I.N., Mamyrova, G., Trieu, E.P., Perurena, O., Koneru, B., O’Hanlon, T.P., Miller, F.W. and Rider, L.G., Childhood Myositis Heterogeneity Study Group, International Myositis Collaborative Study Group (2006) A Novel Autoantibody to a 155-kd Protein Is Associated with Dermatomyositis. Arthritis \& Rheumatology, 54, 3682-3689. http://dx.doi.org/10.1002/art.22164

[6] Kaji, K., Fujimoto, M., Hasegawa, M., Kondo, M., Saito, Y., Komura, K., Matsushita, T., Orito, H., Hamaguchi, Y., Yanaba, K., Itoh, M., Asano, Y., Seishima, M., Ogawa, F., Sato, S. and Takehara, K. (2007) Identification of a Novel Autoantibody Reactive with 155 and 140 kDa Nuclear Proteins in Patients with Dermatomyositis: An Association with Malignancy. Rheumatology (Oxford), 46, 25-28. http://dx.doi.org/10.1093/rheumatology/kel161

[7] Sato, S., Hirakata, M., Kuwana, M., Suwa, A., Inada, S., Mimori, T., Nishikawa, T., Oddis, C.V. and Ikeda, Y. (2005) Autoantibodies to a 140-kd Polypeptide, CADM-140, in Japanese Patients with Clinically Amyopathic Dermatomyositis. Arthritis \& Rheumatology, 52, 1571-1576. http://dx.doi.org/10.1002/art.21023

[8] Sato, S., Hoshino, K., Satoh, T., Fujita, T., Kawakami, Y. and Kuwana M. (2009) RNA Helicase Encoded by Melanoma Differentiation-Associated Gene 5 Is a Major Autoantigen in Patients with Clinically Amyopathic Dermatomyositis: Association with Rapidly Progressive Interstitial Lung Disease. Arthritis \& Rheumatology, 60, 2193-2200. http://dx.doi.org/10.1002/art.24621

[9] Targoff, I.N. (1990) Autoantibodies to Aminoacyl-Transfer RNA Synthetases for Isoleucine and Glycine. Two additional synthetases are antigenic in myositis. The Journal of Immunology, 144, 1737-1743.

[10] Hirakata, M., Mimori, T., Akizuki, M., Craft, J., Hardin, J.A. and Homma, M. (1992) Autoantibodies to Small Nuclear and Cytoplasmic Ribonucleoproteins in Japanese Patients with Inflammatory Muscle Disease. Arthritis \& Rheumatology, 35, 449-456. http://dx.doi.org/10.1002/art.1780350415

[11] Bohan, A. and Peter, J.B. (1975) Polymyositis and Dermatomyositis. The New England Journal of Medicine, 292, 344347. http://dx.doi.org/10.1056/NEJM197502132920706

[12] Lane, R.J., Emslie-Smith, A., Mosquera, I.E. and Hudgson, P. (1989) Clinical, Biochemical and Histological Responses to Treatment in Polymyositis: A Prospective Study. Journal of the Royal Society of Medicine, 82, 333-338.

[13] Sontheimer, R.D. (2002) Would a New Name Hasten the Acceptance of Amyopathic Dermatomyositis (Dermatomyo- 
sitis Sine Myositis) as a Distinctive Subset within the Idiopathic Inflammatory Dermatomyopathies Spectrum of Clinical Illness? Journal of the American Academy of Dermatology, 46, 626-636. http://dx.doi.org/10.1067/mjd.2002.120621

[14] Sato, S., Kuwana, M. and Hirakata, M. (2007) Clinical Characteristics of Japanese Patients with Anti-OJ (Anti-Isoleucyl-tRNAsynthetase) Autoantibodies. Rheumatology (Oxford), 46, 842-845. http://dx.doi.org/10.1093/rheumatology/kel435

[15] Hamaguchi, Y., Kuwana, M., Hoshino, K.,Hasegawa, M., Kaji, K., Matsushita, T., Komura, K., Nakamura, M., Kodera, M., Suga, N., Higashi, A., Ogusu, K., Tsutsui, K., Furusaki, A., Tanabe, H., Sasaoka, S., Muro, Y., Yoshikawa, M., Ishiguro, N., Ayano, M., Muroi, E., Fujikawa, K., Umeda, Y., Kawase, M., Mabuchi, E., Asano, Y., Sodemoto, K., Seishima, M., Yamada, H., Sato, S., Takehara, K. and Fujimoto, M. (2011) Clinical Correlations with Dermatomyositis-Specific Autoantibodies in adult Japanese Patients with Dermatomyositis: A Multicenter Cross-Sectional Study. Archives of Dermatology, 147, 391-398. http://dx.doi.org/10.1001/archdermatol.2011.52

[16] Ikeda, N., Takahashi, K., Yamaguchi, Y., Inasaka, M., Kuwana, M. and Ikezawa, Z. (2011) Analysis of Dermatomyositis-Specific Autoantibodies and Clinical Characteristics in Japanese Patients. The Journal of Dermatology, 38, 973979. http://dx.doi.org/10.1111/j.1346-8138.2011.01262.x

\section{Abbreviations}

PM: polymyositis; DM: dermatomyositis; CADM: clinically amyopathicdermatomyositis; ARS: aminoacyl transfer RNA synthetase; TIF1- $\gamma$ : transcriptional intermediary factor 1- $\gamma$; MDA5: melanoma differentiation-associated gene 5; ILD: interstitial lung disease; RP-ILD: rapidly progressive interstitial lung disease; IP: immunoprecipitation; MMT: manual muscle test; CK: creatine kinase. 